\title{
Meta-analysis of associations between MDM2 SNP309 polymorphism and gastric cancer risk
}

\author{
WEIFENG CHEN $^{1}$, QIANLAN WU ${ }^{2}$ and HONGBO REN ${ }^{1}$ \\ ${ }^{1}$ Department of Neurosurgery, Central Hospital of Handan, Handan, Hebei 056000; ${ }^{2}$ Graduate School of \\ Guangxi Medical University, Nanning, Guangxi Zhuang Autonomous Region 530021, P.R. China
}

Received April 23, 2013; Accepted September 19, 2013

DOI: 10.3892/br.2013.181

\begin{abstract}
Epidemiological studies have been conducted to evaluate genetic variations of murine double minute 2 (MDM2) SNP309 associated with the risk of gastric cancer (GC), although evidence remains conflicting. To gain a better understanding of this relationship, a meta-analysis was performed. Several electronic databases were searched up to February 2013, in order to identify relevant case-control studies. Seven published case-control studies with 2,199 cases and 3,201 controls were included. In the overall analysis, significant associations between the MDM2 SNP309 variant and $\mathrm{GC}$ risk were found for $\mathrm{G}$ vs. T alleles $(\mathrm{OR}=1.35$; $95 \% \mathrm{CI}$, 1.24-1.47), GG vs. TT (OR=1.88; 95\% CI, 1.59-2.24), recessive model (OR=1.71; 95\% CI, 1.49-1.96). Furthermore, stratified by cancer site, significant associations were observed in gastric cardia cancer for all the models, although no significant association was found in any of the models among non-gastric cardia cancer, with the exception of the recessive model. In the subgroup analysis by source of control, MDM2 SNP309 was associated with increased GC risk for the hospital-based case-control (HCC) study for the GG vs. TT, recessive model and for the population-based case-control (PCC) study for the GG vs. TT, recessive model. Following stratification by gender and infection status of Helicobacter pylori (HP) for the recessive model, a significant association was found only in the HP-positive infected individuals. However, no statistically significant association was observed in males, females or the HP-negative infected individuals. In summary, the association between MDM2 SNP309 and GC risk was statistically significant, particularly in gastric cardia cancer for the HP-positive population group.
\end{abstract}

Correspondence to: Dr Weifeng Chen, Department of Neurosurgery, Central Hospital of Handan, 15 Zhonghua South Street, Handan, Hebei 056000, P.R. China

E-mail: chenwf120@gmail.com

Key words: murine double minute 2, gastric cancer, polymorphism, meta-analysis

\section{Introduction}

Gastric cancer (GC) is the fourth most common malignancy and the second primary cause of cancer mortality, resulting in $>800,000$ deaths worldwide annually (1-3). The overall prognosis for patients with GC remains poor and the 5-year survival rates range from 10 to $28 \%$ (4). The mechanisms that result in the tumorigenesis and progression of $\mathrm{GC}$ remain to be elucidated, but etiological factors include interactions between genetic and environmental factors.

Murine double minute 2 (MDM2) was first described as one of the genes amplified on the double minute chromosomes present in the spontaneously transformed Balb/c 3T3-DM cells in culture (5). The amplified sequences deriving from chromosome 10, region C1-C3. NIH3T3 or Rat 2 cells overexpressing only the cloned MDM2 gene were tumorigenic in nude mice, revealing that MDM2 itself possesses transforming activity (6). Tp53 gene, which plays an important role in oncogenesis, inhibits cell proliferation and promotes cell apoptosis. However, MDM2 and p53 maintain an antagonistic relationship (7). TP53 is a transcription factor activated by cell stress conditions, such as hypoxia, DNA damage, and oncogene activation. Its downstream gene MDM2 is also activated. TP53 causes intracellular expression of MDM2, while the latter leads to the downregulation of TP53, which is the autoregulatory feedback loop between TP53 and MDM2 $(8,9)$. MDM2 is known to regulate the function of p53 in different ways (7). It combines directly to p53 to inhibit TP53-dependent transcription, prevent the interaction of p53 and its transcription factors, and also mediate the transport of the MDM2-combined p53 from nucleus to cytoplasm.

MDM2 SNP309 is a single nucleotide $\mathrm{T}$ to $\mathrm{G}$ polymorphism located at the 309th nucleotide in the MDM2 gene promoter. Over the last two decades, it has been confirmed that the MDM2 SNP309 polymorphism is capable of significantly altering the expression of MDM2, thereby suppressing the p53 pathway. Moreover, a number of epidemiological studies have investigated the association between MDM2 SNP309 and the risk of various types of cancer (10-13), including renal, hepatocellular, endometrial, bladder and gastric. However, results concerning the relationship between MDM2 SNP309 polymorphism and GC risk were different or even contradictory $(14,15)$. This difference or contradiction in results may be due to the relatively small sample size of individual studies and interactions of gene and environmental factors during gastric tumorigenesis. 
In the present study, we examined the association between the MDM2 SNP309 polymorphism and GC risk, and performed a meta-analysis of 7 case-control studies including 2,199 cases and 3,201 controls focusing on overall and subgroup factors.

\section{Materials and methods}

Literature search. Studies published prior to February, 2013 were identified through a systematic search of the following electronic databases: PubMed, MEDLINE, Embase, Web of Science, China Biology Medicine disc, using key words including: 'MDM2' or 'Murine double minute 2' and 'polymorphism' or 'variant' and 'gastric' or 'stomach' and 'neoplasm' or 'tumor' or 'cancer' or 'carcinoma', without restriction on language. Additionally, an extensive manual search was performed by using references from each retrieved study or review article.

Inclusion criteria. The eligible studies were required to meet the following criteria: i) case-control studies evaluating the association between MDM2 SNP309 and GC risk, and ii) supply detailed data of the genotype distribution of the MDM2 SNP309 polymorphisms in cases and controls in order to estimate the OR with $95 \%$ CI. When authors reported on the same patient populations in two or more publications, only the most recent or complete study was included in the review, in order to avoid overlapping between studies.

Data extraction. Two authors (Q.W. and W.C.) independently extracted the following data from each included study by using a standardized form: the first author, publication year, country of origin, characteristics of controls, sources of controls, genotyping method, and genotype distribution. If there was any inconsistency, it was resolved among all the authors. When essential data were not presented in studies, every effort was made to contact the corresponding authors.

Statistical methods. Overall and subgroup meta-analyses were performed with stratification by gender, cancer location, infection status of Helicobacter pylori (HP) or source of controls. Pooled ORs and 95\% CIs were calculated to evaluate the association between MDM2 SNP309 and GC risk. Five genetic models [G/T; GG/TT; GT/TT; the dominant model, i.e., (GG+GT)/TT; and the recessive model, i.e., GG/(TT+GT)] and the mathematical models, fixed- and random-effects models, were applied to obtain a more comprehensive analysis. The heterogeneity test was used to determine which mathematical model was more suitable. The fixed-effects model was applied when the Chi-square-based Q-test yielded $\mathrm{P}>0.05, \mathrm{I}^{2} \leq 50 \%$, otherwise, the random-effects model was applied. In case of any heterogeneity $\left(\mathrm{P}<0.05, \mathrm{I}^{2}>50 \%\right)$, sensitivity analyses were carried out to explore possible explanations for the heterogeneity included in the meta-analysis. Publication bias was not assessed using tests for funnel plot asymmetry as there were fewer than nine studies (16). Calculations were performed using The Cochrane Collaboration's software RevMan 5.20.

\section{Results}

Characteristics of the various studies. The search for eligible studies yielded 10 references that assessed the relationship of
MDM2 SNP309 and GC risk, of which one article (14) was excluded because it was conducted on overlapping populations with another included study (13) (the study including the larger sample number). The study by Pan et al (17), which included the meta-analysis results of five studies, was excluded to avoid overlapping with the study by Zhang et al (13). The study by $\mathrm{Li}$ (18) was excluded as it did not provide the relevant genotype frequencies. Two of the 10 studies $(19,20)$ were confirmed to have been conducted on different populations from different time periods after the author was contacted and were included in the present meta-analysis. Seven case-control studies $(13,15,19-23)$ published from 2006 to 2012 and including 2,199 cases and 3,201 controls, explored the association between the relationship between MDM2 SNP309 and GC risk. The studies focused on Asian populations. Of the seven publications, five studies $(13,15,21-23)$ were published in English, and two in Chinese $(19,20)$. The Hardy-Weinberg equilibrium (HWE) test was performed on all the included studies except that by Yang et al (22) in which HWE was not reported. The remaining studies were shown to be in HWE ( $>0.05)$, with the except of that by Er et al (20). The main characteristics of the seven studies are shown in Table I.

Meta-analysis. As shown in Fig. 1, we found significant associations between the MDM2 SNP309 polymorphism and GC susceptibility in the overall analysis for the three models including $\mathrm{G}$ vs. T alleles, GG vs. TT, and the recessive model (for G vs. T: OR=1.35 and 95\% CI, 1.24-1.47; for GG vs. TT: $\mathrm{OR}=1.88$ and $95 \% \mathrm{CI}, 1.59-2.24$, and the recessive model: $\mathrm{OR}=1.71$ and $95 \% \mathrm{CI}, 1.49-1.96$. However, no association was found between the two models (GT vs. TT and dominant model) and GC risk (for GT vs. TT: OR=1.08 and 95\% CI, $0.83-1.42$, and the dominant model: $\mathrm{OR}=1.26$ and $95 \% \mathrm{CI}$, 0.96-1.66).

In the stratified analysis by cancer location, significantly increased risks were found among gastric cardia cancer (GCA) (999 cases and 2,322 controls) for all the models (for GG vs. TT: OR=2.00 and 95\% CI, 1.61-2.50; for GT vs. TT: $\mathrm{OR}=1.50$ and $95 \% \mathrm{CI}, 1.20-1.88$; the dominant model: $\mathrm{OR}=1.70$ and 95\% CI, 1.38-2.10; and the recessive model: $\mathrm{OR}=1.63$ and 95\% CI, 1.38-1.94). Among non-GCA (1,200 cases and 1,453 controls), no significant association was found in GG vs. TT $(\mathrm{OR}=1.45$ and $95 \% \mathrm{CI}, 0.88-2.39)$, GT vs. TT: $\mathrm{OR}=0.83$ and $95 \% \mathrm{CI}=0.69-1.00$, and the dominant model: $\mathrm{OR}=0.99$ and 95\% CI, 0.83-1.18. However, significantly elevated risk was associated with the recessive model $(\mathrm{OR}=1.86$ and $95 \% \mathrm{CI}$, 1.51-2.29) (Table II)

In the subgroup analysis by source of controls, for the hospital-based case-control (HCC) study, there was little evidence that the MDM2 SNP309 resulted in an elevated risk for GT vs. TT $(\mathrm{OR}=1.12$ and $95 \% \mathrm{CI}, 0.68-1.84)$ and the dominant model (OR=1.35 and 95\% CI, 0.83-2.20). However, the association was observed with $\mathrm{GG}$ vs. TT $(\mathrm{OR}=1.91$ and 95\% CI, 1.12-3.25) and the recessive model $(\mathrm{OR}=1.55$ and 95\% CI, 1.17-2.04). For the population-based case-control (PCC) study, no association was found with any of the genetic models with the exception of GG vs. TT (Table II).

In the included studies, two studies $(13,15)$ comprising 813 cases and 873 controls provided separate raw data of genotypic distribution for the recessive model for an analysis 

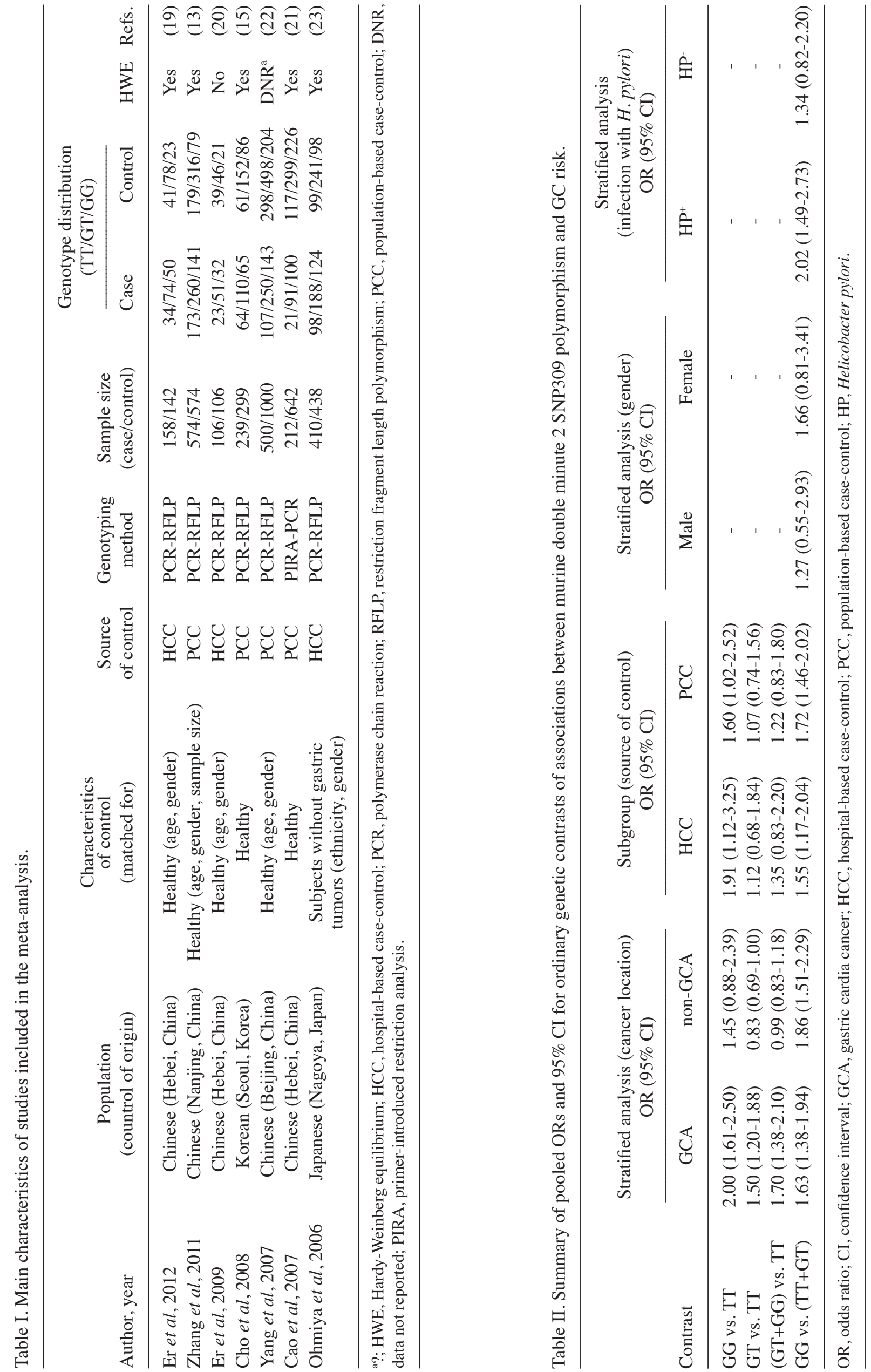


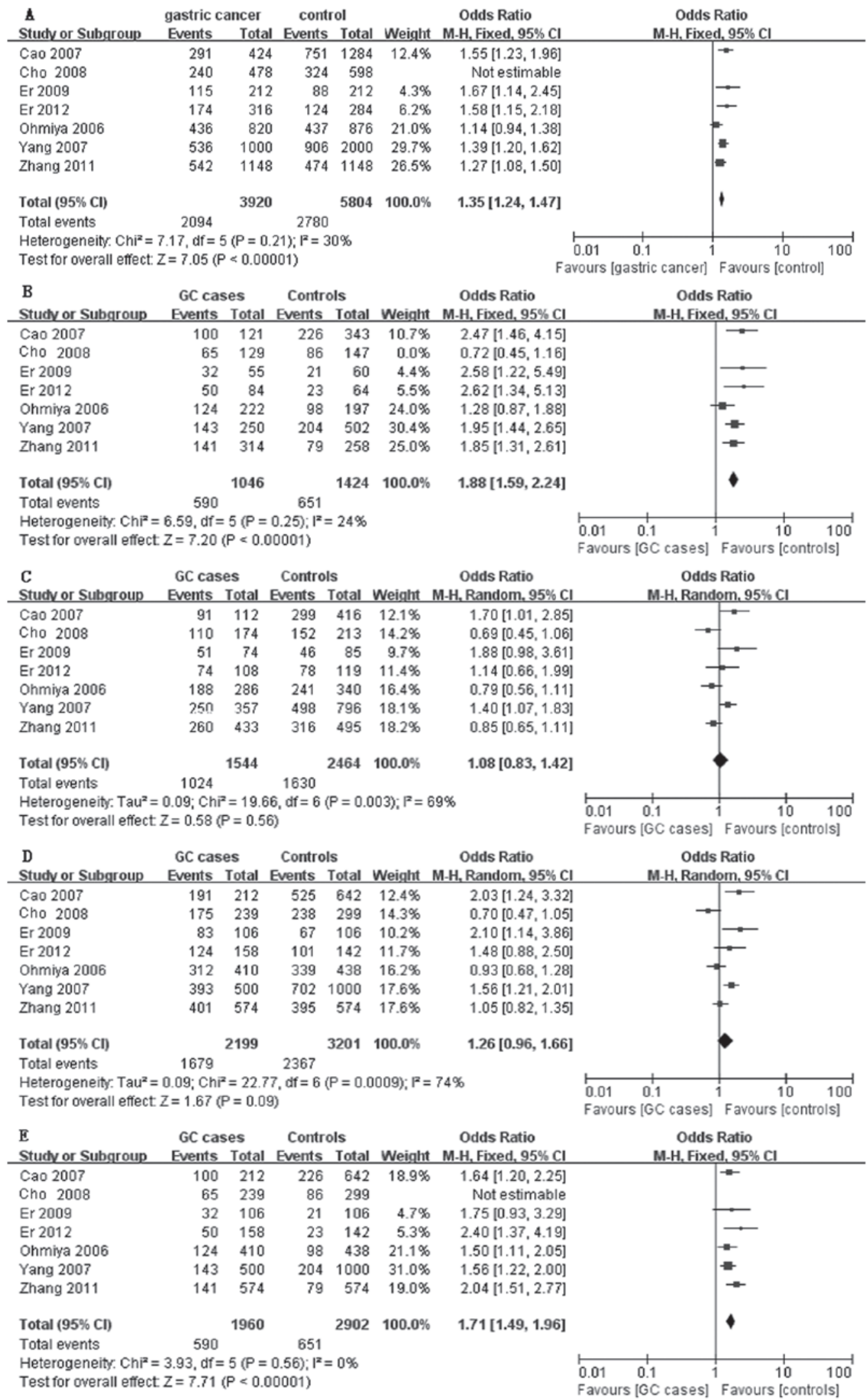

Figure 1. Forest plot shows the association between murine double minute 2 (MDM2) SNP309 and gastric cancer risk: (A) G vs. T allele, (B) GG vs. TT, (C) GT vs. TT, (D) (GT+GG) vs. TT, (E) GG vs. (TT+GT).

of gender stratification, but no effect on the MDM2 SNP309 polymorphism on GC susceptibility was observed for males $(\mathrm{OR}=1.27$ and $95 \% \mathrm{CI}, 0.55-2.93)$ or females $(\mathrm{OR}=1.66$ and
95\% CI, 0.81-3.41). The pooled ORs and their 95\% CIs are presented in Table II, and the forest plot of the stratified analyses was plotted in Fig. 2. 


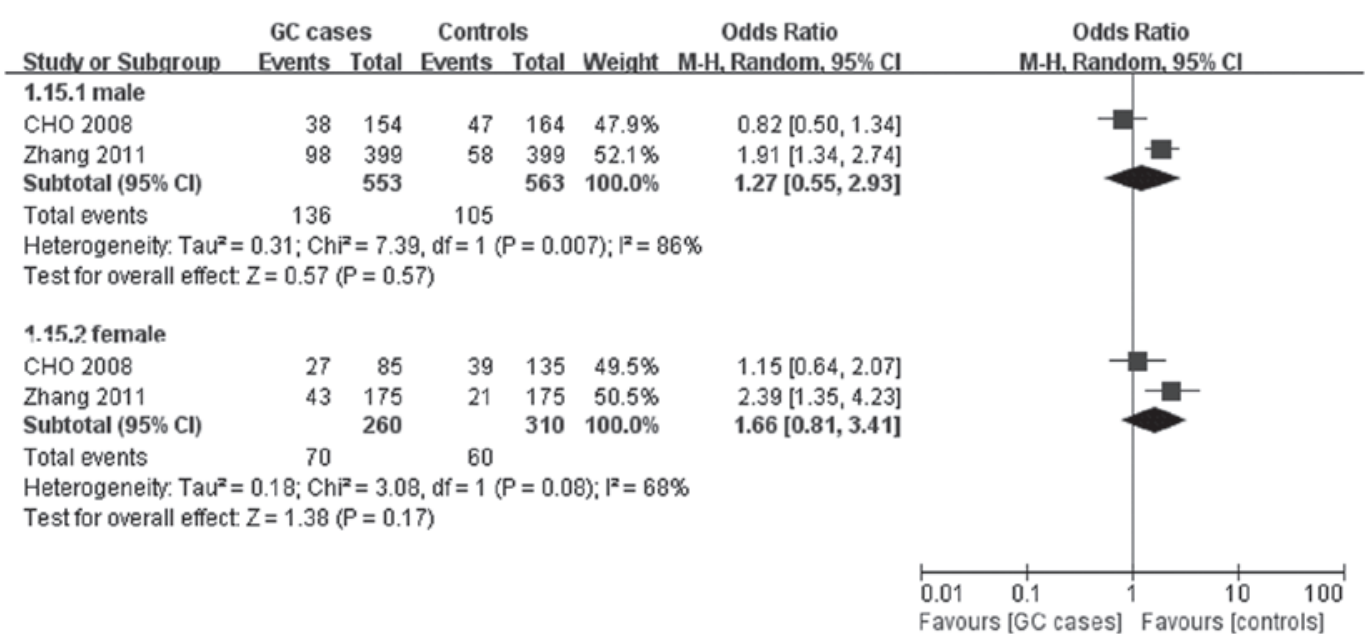

Figure 2. Meta-analysis for the association of gastric cancer risk with murine double minute 2 SNP309 polymorphism (recessive model; stratified by gender).

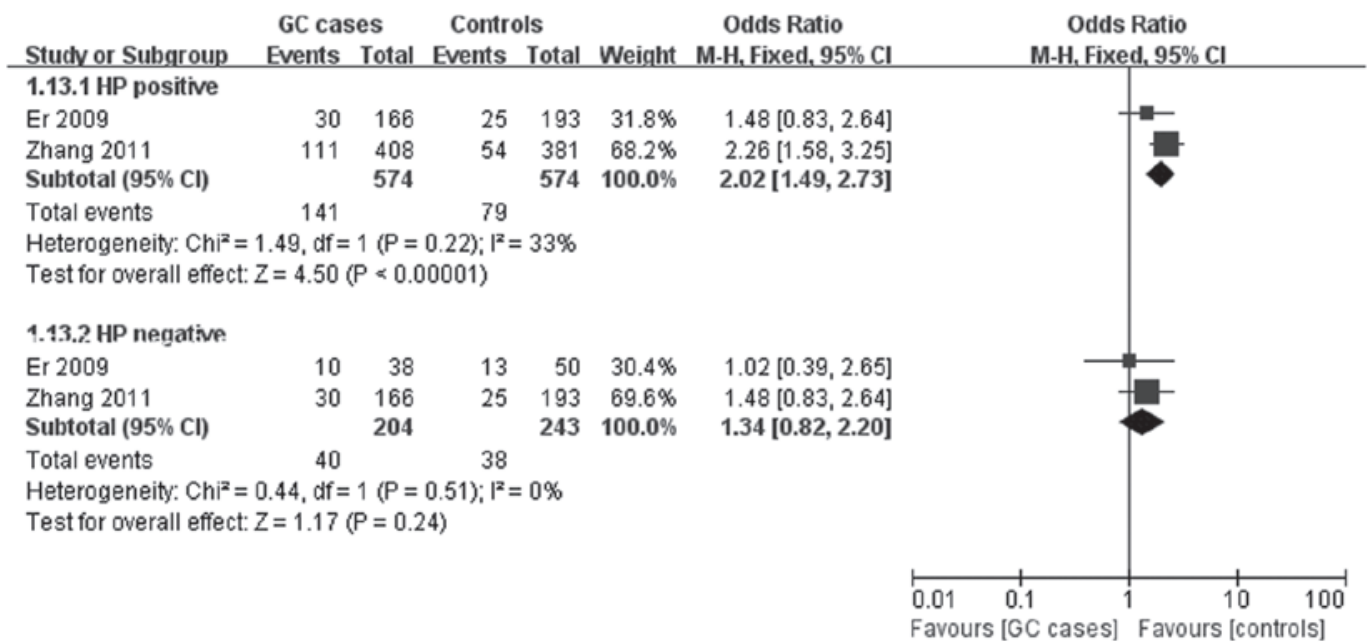

Figure 3. Meta-analysis for the association of gastric cancer risk with murine double minute 2 SNP309 polymorphism (recessive model; stratified by infection status of Helicobacter pylori).

Two studies $(13,20)$ provided detailed data of the genotype distribution of the recessive model for a stratified analysis of HP infection. An association was found with HP infection (for $\mathrm{HP}^{+}$: OR=2.02 and 95\% CI, 1.49-2.73), but not with non-infection of HP (for HP: OR=1.34 and 95\% CI, 0.82-2.20). The pooled ORs and their 95\% CIs are presented in Table II, and the forest plot of the stratified analyses is shown in Fig. 3.

Sensitivity analysis. Sensitivity analysis was performed by deleting a single study involved each time to reflect the effect of the individual data on the pooled ORs. In both the overall and subgroup analyses, the corresponding pooled results were not markedly affected for all the models, except for the stratified analysis by cancer location for GCA GT vs. TT (data not shown).

\section{Discussion}

It is well known that risk factors of GC involve HP infection, smoking, alcohol, diet and nutrition such as red and processed meat, genetics and epigenetic factors (24). Additionally, host genetic susceptibility has been confirmed to play an important role in the development of GC. In the last decade, a number of meta-analyses have demonstrated that the MDM2 SNP309 G allele contributes to an increased risk of various human malignancies (25-29), such as liver, ovarian, head and neck, and lung cancer. Some meta-analyses also revealed that MDM2 SNP309 polymorphism may exert a differential effect on cancer risk along with ethnicity, including breast cancer and head and neck squamous cell carcinoma (HNSCC) $(30,31)$. The present meta-analysis was conducted to examine a comprehensive evaluation regarding the association of MDM2 SNP 309 polymorphism with GC.

Findings of the overall analysis demonstrated that significant associations between the MDM2 SNP309 variant and $\mathrm{GC}$ risk were identified for the $\mathrm{G}$ vs. T alleles, GG vs. TT, and recessive model. It clearly revealed that $\mathrm{G}$ allele acts a key factor to increase the risk of GC, which is in accordance with Pan et al (17).

Since the interaction between genotype and environment acts a major determinant of tumor risk (32), we also conducted stratified analyses for GC risk based on cancer location, 
gender, infection status of HP, and a subgroup analysis by source of control.

Stratified analysis by cancer location revealed that increased risks with the MDM2 SNP309 variant for all the genotype models were more pronounced among GCA patients, which was contradictory to the results of Zhang et al (13), but in accordance with Yang et al (22). The latter authors found that a significantly increased risk for developing GCA was associated with the MDM2 $309 \mathrm{G}$ allele and the P53 72 Pro allele compared with the MDM2 $309 \mathrm{~T}$ allele and the P53 72 Arg allele in an allele dose-dependent manner. Moreover, Yang et al (22) identified a joint effect between the MDM2 and P53 polymorphisms in intensifying GCA risk. This finding may due to the differences between GCA and GC at other sites with regard to epidemiological characteristics, etiology, pathogenesis and clinical behavior.

Recent meta-analyses have also found that SNP309G is associated with increasing lung cancer risk in females but not in males $(29,33)$. However, in the stratified analysis by gender, we did not observe any evidence of their interaction in all the genotype models. In addition, in the seven eligible studies, two studies $(21,22)$ reported detailed data concerning stratified analysis by smoking, and another two studies $(13,15)$ analysed the association between age and GC risk by stratified analysis. However, we were not able to combine the data since the diagnostic criteria of smoking and non-smoking and the cut-off age were inconsistent or different.

An association was found with infection of HP but not with non-infection of HP in the stratified analysis by infection status of HP. Kodama et al (34) found that successful eradication of HP markedly decreased the p53 and MDM2 levels. Therefore, authors of that study suggested that HP infection may be associated with alteration of p53 pathway-mediated cell proliferation and apoptosis and correlate with MDM2 polymorphism via the p53 pathway. Their hypothesis may strongly enhance our stratified analysis results.

Although a careful search for publications, using explicit criteria for study inclusion, precise data extraction, and strict data analysis was conducted, the present meta-analysis had a number of limitations. Firstly, the small number of available studies and participants included, and certain factors including smoking, age and prognosis were not evaluated for lack of raw data. Secondly, the heterogeneity of some endpoints may have limited the comparability among studies and affected the validity of our results, which potentially result from differences in the selection of controls, age distribution and prevalence of lifestyle factors. Thirdly, while no publication bias was identified, our results may have been biased towards positive results as negative results were not yielded. To confirm our findings, well-designed studies with large samples are required in the future.

In summary, results of this meta-analysis suggest that the G allele of MDM2 SNP309 is a high-risk factor for developing GC. Furthermore, this phenomenon was more notable in subgroups including the GCA and infection with HP population groups. However, large-scale studies using standardized unbiased methods, well-matched controls and estimating the effect of gene-gene and gene-environment interactions should be used to provide a better, comprehensive understanding of the relationship of MDM2 SNP309 and GC risk.

\section{References}

1. Nakao M, Matsuo K, Ito $\mathrm{H}$, et al: ABO genotype and the risk of gastric cancer, atrophic gastritis, and Helicobacter pylori infection. Cancer Epidemiol Biomarkers Prev 20: 1665-1672, 2011.

2. Duell EJ, Travier N, Lujan-Barroso L, et al: Alcohol consumption and gastric cancer risk in the European Prospective Investigation into Cancer and Nutrition (EPIC) cohort. Am J Clin Nutr 94: 1266-1275, 2011.

3. Jemal A, Center MM, DeSantis C and Ward EM: Global patterns of cancer incidence and mortality rates and trends. Cancer Epidemiol Biomarkers Prev 19: 1893-1907, 2010.

4. Ebert MP, Niemeyer D, Deininger SO, et al: Identification and confirmation of increased fibrinopeptide a serum protein levels in gastric cancer sera by magnet bead assisted MALDI-TOF mass spectrometry. J Proteome Res 5: 2152-2158, 2006.

5. Cahilly-Snyder L, Yang-Feng T, Francke U and George DL: Molecular analysis and chromosomal mapping of amplified genes isolated from a transformed mouse 3T3 cell line. Somat Cell Mol Genet 13: 235-244, 1987.

6. Fakharzadeh SS, Trusko SP and George DL: Tumorigenic potential associated with enhanced expression of a gene that is amplified in a mouse tumor cell line. EMBO J 10: 1565-1569, 1991.

7. Piette J, Neel H and Marechal V: Mdm2: keeping p53 under control. Oncogene 15: 1001-1010, 1997.

8. Chen H, Xie L and Liu B: Clinical significance of MDM2 as a tumor biomarker. Chin-Ger J Clin Oncol 11: 356-360, 2012.

9. Picksley SM and Lane DP: The p53-mdm2 autoregulatory feedback loop: a paradigm for the regulation of growth control by p53? Bioessays 15: 689-690, 1993.

10. Hirata H, Hinoda Y, Kikuno N, et al: MDM2 SNP309 polymorphism as risk factor for susceptibility and poor prognosis in renal cell carcinoma. Clin Cancer Res 13: 4123-4129, 2007.

11. Terry K, McGrath M, Lee IM, Buring J and De Vivo I: MDM2 SNP309 is associated with endometrial cancer risk. Cancer Epidemiol Biomarkers Prev 17: 983-986, 2008.

12. Olsson H, Hultman P, Rosell J, Söderkvist P and Jahnson S MDM2 SNP309 promoter polymorphism and p53 mutations in urinary bladder carcinoma stage T1. BMC Urol 13: 5, 2013.

13. Zhang GX, Li YQ and Pan XL: Polymorphism of MDM2 promoter, regulated by helicobacter pylori lipopolysaccharide, is associated with both an increased susceptibility to gastric carcinoma and poor prognosis in Chinese patients. J Gastroenterol Hepatol 26: 280, 2011.

14. Wang X, Yang J, Ho B, et al: Interaction of Helicobacter pylori with genetic variants in the MDM2 promoter, is associated with gastric cancer susceptibility in Chinese patients. Helicobacter 14: 114-119, 2009.

15. Cho YG, Choi BJ, Song JH, et al: No association of MDM2 T309G polymorphism with susceptibility to Korean gastric cancer patients. Neoplasma 55: 256-260, 2008.

16. Egger M and Smith GD: Bias in location and selection of studies. BMJ 316: 61-66, 1998

17. Pan X, Li Y, Feng J, et al: A functional polymorphism T309G in MDM2 gene promoter, intensified by Helicobacter pylori lipopolysaccharide, is associated with both an increased susceptibility and poor prognosis of gastric carcinoma in Chinese patients. BMC Cancer 13: 126, 2013.

18. Li Z: Association of gene polymorphisms with helicobater pylori related gastric cancer in a Chinese population. (unpublished masters thesis). 2011.

19. ER L, Zhang L, Niu W, et al: Relevance of MDM2 polymorphisms with esophageal squamous cell carcinoma, gastric adenocarcinoma and double primary cancers in esophagus and stomach. Mod Prev Med 39: 3342, 2012.

20. Er L, Zhang L, Niu W, et al: Relation of MDM2 gene polymorphism and Helicobacter pylori infection to gastric cardiac carcinoma. Clin Focus 24: 1594-1597, 2009.

21. Cao YY, Zhang XF, Guo W, Wang R, Ge H and Zhang JH: Association of the MDM2 polymorphisms with susceptibility of esophageal squamous cell carcinoma and that of gastric cardiac adenocarcinoma. Tumor 27: 628-632, 2007.

22. Yang M, Guo Y, Zhang X, et al: Interaction of P53 Arg72Pro and MDM2 T309G polymorphisms and their associations with risk of gastric cardia cancer. Carcinogenesis 28: 1996-2001, 2007.

23. Ohmiya N, Taguchi A, Mabuchi N, et al: MDM2 promoter polymorphism is associated with both an increased susceptibility to gastric carcinoma and poor prognosis. J Clin Oncol 24: 4434-4440, 2006. 
24. González CA and Agudo A: Carcinogenesis, prevention and early detection of gastric cancer: Where we are and where we should go. Int J Cancer 15: 745-753, 2012.

25. Bond GL, Menin C, Bertorelle R, Alhopuro P, Aaltonen LA and Levine AJ: MDM2 SNP309 accelerates colorectal tumour formation in women. J Med Genet 43: 950-952, 2006.

26. Ma HB, Huang T, Han F and Chen WY: Association between MDM2 promoter SNP309 T/G polymorphism and liver cancer risk - a meta-analysis. Asian Pac J Cancer Prev 13: 2841-2846, 2012.

27. Ma YY, Guan TP, Yao HB, et al: The MDM2 309T>G polymorphism and ovarian cancer risk: a meta-analysis of 1534 cases and 2211 controls. PLoS One 8: e55019, 2013.

28. Zhang Y, Bai Y, Zhang Y, Guan J and Chen L: The MDM2 309 $\mathrm{T} / \mathrm{G}$ polymorphism is associated with head and neck cancer risk especially in nasopharyngeal cancer: a meta-analysis. Onkologie 35: 666-670, 2012.

29. Zhuo W, Zhang L, Zhu B, Ling J and Chen Z: Association of MDM2 SNP309 variation with lung cancer risk: evidence from 7196 cases and 8456 controls. PLoS One 7: e41546, 2012.
30. Liu J, Zheng Y, Lei D, et al: MDM2 309T>G polymorphism and risk of squamous cell carcinomas of head and neck: a meta-analysis. Asian Pac J Cancer Prev 12: 1899-1903, 2011.

31. Economopoulos KP and Sergentanis TN: Differential effects of MDM2 SNP309 polymorphism on breast cancer risk along with race: a meta-analysis. Breast Cancer Res Treat 120: 211-216, 2010.

32. Wiseman M: The Second World Cancer Research Fund/American Institute for Cancer. Proc Nutr Soc 67: 253-256, 2008

33. He W, Long J, Xian L, et al: MDM2 SNP309 polymorphism is associated with lung cancer risk in women: a meta-analysis using METAGEN. Exp Ther Med 4: 569-576, 2012.

34. Kodama M, Fujioka T, Murakami K, et al: Eradication of Helicobacter pylori reduced the immunohistochemical detection of p53 and MDM2 in gastric mucosa. J Gastroenterol Hepatol 20: 941-946, 2005. 